\title{
Defect-Induced Orbital Polarization and Collapse of Orbital Order in Doped Vanadium Perovskites
}

\author{
Adolfo Avella, ${ }^{1,2,3}$ Andrzej M. Oleś, ${ }^{4,5}$ and Peter Horsch ${ }^{5}$ \\ ${ }^{1}$ Dipartimento di Fisica “E.R. Caianiello”, Università degli Studi di Salerno, I-84084 Fisciano (SA), Italy \\ ${ }^{2}$ CNR-SPIN, UOS di Salerno, I-84084 Fisciano (SA), Italy \\ ${ }^{3}$ Unità CNISM di Salerno, Università degli Studi di Salerno, I-84084 Fisciano (SA), Italy \\ ${ }^{4}$ Marian Smoluchowski Institute of Physics, Jagiellonian University, Prof. S. Łojasiewicza 11, PL-30348 Kraków, Poland \\ ${ }^{5}$ Max-Planck-Institut für Festkörperforschung, Heisenbergstrasse 1, D-70569 Stuttgart, Germany
}

(Dated: 21 January 2019)

\begin{abstract}
We explore mechanisms of orbital-order decay in the doped Mott insulators $R_{1-x}(\mathrm{Sr}, \mathrm{Ca})_{x} \mathrm{VO}_{3}(R=\mathrm{Pr}, \mathrm{Y}, \mathrm{La})$ caused by charged $(\mathrm{Sr}, \mathrm{Ca})$ defects. Our unrestricted Hartree-Fock analysis focuses on the combined effect of random charged impurities and associated doped holes up to $x=0.5$. The study is based on a generalized multi-band Hubbard model for the relevant vanadium $t_{2 g}$ electrons, and includes the long-range (i) Coulomb potentials of defects and (ii) electron-electron interactions. We show that the rotation of $t_{2 g}$ orbitals, induced by the electric field of defects, is a very efficient perturbation that largely controls the suppression of orbital order in these compounds. We investigate the inverse participation number spectra and find that electron states remain localized on few sites even in the regime where orbital order is collapsed. From the change of kinetic and superexchange energy we can conclude that the motion of doped holes, which is the dominant effect for the reduction of magnetic order in high- $T_{c}$ compounds, is of secondary importance here.
\end{abstract}

Doping of Mott insulators is a central topic in materials science [1, 2], cold gases [3], and many-body theory [4] — firstly because of the intriguing origin of the insulating state, due to strong electron correlations, and secondly owing to the amazing features that can emerge when they are doped, such as superconductivity in cuprates [5]-10], magneto- and thermoelectric effects in manganites [11-15] and heterostructures [16-19]. Yet, often such systems remain insulating when doped, although transitions into metallic or superconducting states were expected [20]. The cubic vanadium perovskites show, despite strong quantum orbital fluctuations [21-25], an unusual gradual decay of orbital and spin order and a not-well-defined crossover into a poor metallic state at high doping $x$, e.g., $x=0.18$ in $\mathrm{La}_{1-x} \mathrm{Sr}_{x} \mathrm{VO}_{3}$ and $x=0.50$ in $\mathrm{Y}_{1-x} \mathrm{Ca}_{x} \mathrm{VO}_{3}$ [26]-29]. This makes them an ideal platform for the study of charged defects and of their interaction with doped holes in systems with spin-orbital degrees of freedom [30, 31].

Vanadates are Mott insulators where the $t_{2 g}$ electrons form a $d^{2}$ configuration with a $S=1$ spin at each $\mathrm{V}$ ion. A small crystal field (CF) lowers the energy of $x y$ orbitals by $\Delta_{c} \simeq 0.1$ $\mathrm{eV}$ with respect to $\{y z, z x\}$ orbital doublet [27,-29, 32-36], which is the source of strong orbital quantum fluctuations [2125]. The breaking of an almost perfect cubic crystal symmetry leads to highly anisotropic electronic states. The undoped systems reveal two distinct spin-orbital ordered ground states. In systems with a large $R$-ion radius, as $\mathrm{LaVO}_{3}$, the ground state has coexisting spin $C$-type $\mathrm{AF}(C$-AF) and $G$-type alternating orbital $(G-\mathrm{AO})$ order [32-36], which is stabilized by the effective spin-orbital superexchange interactions [21, 37]. A second type of complementary $G$-AF/C-AO spin-orbital order results from a competition of superexchange and JahnTeller (JT) interactions [21] and occurs in undoped $R \mathrm{VO}_{3}$ perovskites with small radii of $R$ ions, as in $\mathrm{YVO}_{3}[32,-36]$.

Motivations to analyze the role of charged defects are: (i) the surprising discovery that the $G-\mathrm{AF} / C$-AO ground state of $\mathrm{YVO}_{3}$ changes already at $x \simeq 1 \% \mathrm{Ca}$ doping into the $C-\mathrm{AF} / G$-AO state [32, 38, 39], and (ii) the stability of the latter phase up to high doping [29, 40, 41]. The fragility of $G$ - $\mathrm{AF} / C$-AO order relative to $C$-AF/ $G$-AO phase was explained by a double exchange process for the doped hole bound to the charged defect, triggered by the FM correlations in the $C$-AF state [42]. Subsequent studies have shown that the holes in the $C$-AF/ $G$-AO state are confined and bound to the charged defects, leading to a gradual decay of order proportional to doping, yet not to its collapse [31].

In this Letter, we investigate the doping dependence of the orbital order (OO) in doped vanadates and explain its collapse. We find that the dominant decay mechanism is the rotation of $t_{2 g}$ electron states induced by the Coulomb potential of defects. This orbital polarization involves all $t_{2 g}$ orbitals at $\mathrm{V}$ ions surrounding the defect [43], i.e., on the defect cube, see Fig. 1. Interestingly, the OO collapse is visible in the moderate delocalization of the states in the upper Hubbard band (UHB) and identified as $d^{2} \rightarrow d^{3}$ high spin transitions at $\mathrm{V}$ ions on the defect cubes.

The Hamiltonian for the $t_{2 g}$ electrons in $R_{1-x} \mathrm{Ca}_{x} \mathrm{VO}_{3}$,

$$
\mathcal{H}_{t 2 g}=\mathcal{H}_{\mathrm{Hub}}+\mathcal{H}_{\mathrm{pol}}+\sum_{i<j} v\left(r_{i j}\right) \hat{n}_{i} \hat{n}_{j}+\sum_{m i} v\left(r_{m i}\right) \hat{n}_{i}
$$

includes the extended degenerate Hubbard model $\mathcal{H}_{\mathrm{Hub}}$ [44], orbital-polarization term $\mathcal{H}_{\mathrm{pol}}$ [43], and two last terms stand for $t_{2 g}$ electron-electron interactions and the repulsive potential of Ca defects. Both are determined by the Coulomb interaction $\propto v(r) \equiv e^{2} / \varepsilon_{c} r$, where $\varepsilon_{c} \simeq 5$ [42] is the dielectric constant of the core electrons, and $r$ is the distance between interacting charges of: (i) two $\mathrm{V}$ ions at sites $i$ and $j$ with $r_{i j}=\left|\mathbf{r}_{i}-\mathbf{r}_{j}\right|$, and (ii) (Ca,Sr) defect at site $m$ and a $t_{2 g}$ electron at a $\mathrm{V}$ ion at site $i$, with $r_{m i}=\left|\mathbf{R}_{m}-\mathbf{r}_{i}\right|$. We emphasize that the latter term acts as a potential from all defects on the $t_{2 g}$ electron charge $\hat{n}_{i}=\sum_{\alpha \sigma} \hat{n}_{i \alpha \sigma}$, with $\hat{n}_{i \alpha \sigma}=\hat{d}_{i \alpha \sigma}^{\dagger} \hat{d}_{i \alpha \sigma}$.

The hopping of the $t_{2 g}$ electrons $\propto t \equiv(d d \pi)$ in $\mathcal{H}_{\mathrm{Hub}}$ is 
(a)

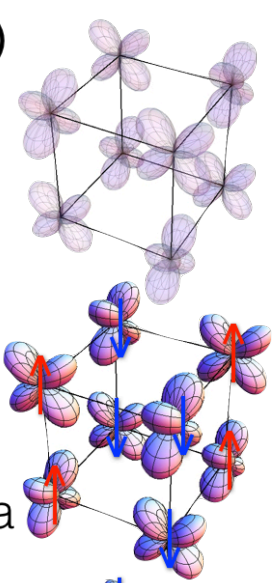

(b)

C

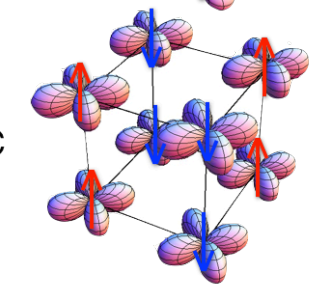

(c) $\overbrace{x_{x}}^{y}$
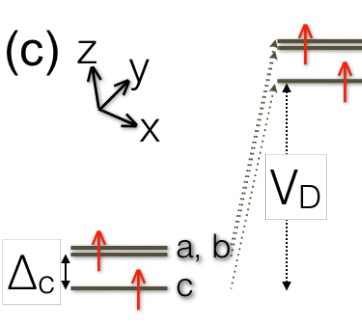

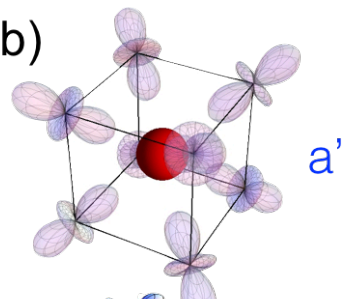

a'
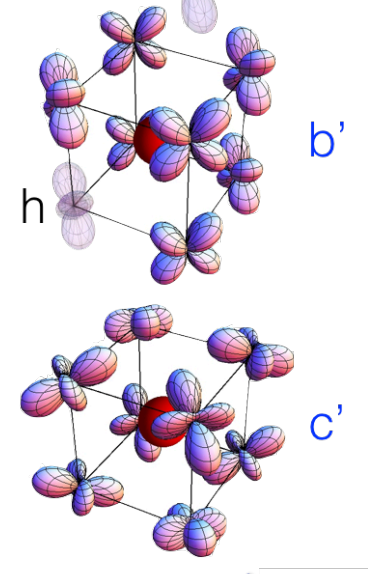

$a, b$
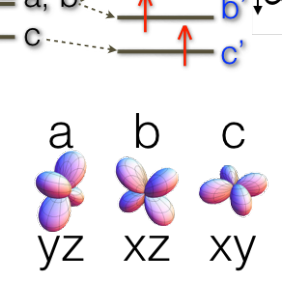

FIG. 1. Schematic view of occupied and unoccupied (grayed out) $t_{2 g}$ V orbitals for: (a) $G$-AO order in undoped $R \mathrm{VO}_{3}$ with $C$-AF spin order marked by red/blue arrows, and (b) a defect cube around a $\mathrm{Ca}^{2+}$ defect (red sphere) in $R_{1-x} \mathrm{Ca}_{x} \mathrm{VO}_{3}$, with $\left\{a^{\prime}, b^{\prime}, c^{\prime}\right\}$ orbitals in the large $\mathcal{D}$ limit. Finite $\mathcal{D}$ modifies the standard $t_{2 g}$ basis $\{a, b, c\}$ at each $\mathrm{V}$ site to $\left\{a^{\prime}, b^{\prime}, c^{\prime}\right\}$; the lowest orbitals $\left\{c^{\prime}, b^{\prime}\right\}$ are occupied at all but the hole (h) site. (c) $t_{2 g}$ orbital energies at a $\mathrm{V}$ ion for $\mathcal{D} \sim \Delta_{c} / 2$, with the $\left\{a^{\prime}, b^{\prime}\right\}$ doublet split by $2 \mathcal{D}$.

two-dimensional and orbital flavor conserving [42-45], which has peculiar consequences for hole propagation [46-50]. Below we denote the $t_{2 g}$ orbitals $\{y z, x z, x y\}$ by the cubic directions $\{a, b, c\}$, respectively, for which the hopping is forbidden [51] (see Fig. 11). Intraatomic Coulomb interactions are parametrized by intraorbital $U$ and Hund's exchange $J_{H}$. The rotational invariant form [52] is essential for multi-orbital models when orbitals and/or spins rotate [43, 53]. The cubic symmetry of the spin-orbital structure is broken by a CF term $\propto \Delta_{c}$, which favors the $c^{1}(a / b)^{1}$ electronic configuration at $\mathrm{V}^{3+}$ ions. The 2 nd electron can select between two degenerate orbitals $\{a, b\}$, according to the spin-orbital superexchange interaction that emerges from the present Hubbard model [21], see Fig. 11(a).

A Ca ${ }^{2+}$ defect in the lattice of $\mathrm{Y}^{3+}$ ions in $\mathrm{Y}_{1-x} \mathrm{Ca}_{x} \mathrm{VO}_{3}$ acts effectively as a negative charge, which repels all vanadium electrons on a defect cube by $V_{\mathrm{D}} \equiv v(d)$, as shown in Fig. 1.c). As we are dealing with a Mott insulator the upward shift creates defect states in the Mott-Hubbard gap [42]. In this work, we focus on another effect of the defect's charge that is displayed in Fig. 11(b). The $t_{2 g}$ vanadium orbitals on a defect cube rotate to reduce their Coulomb energy in the electric field of the defect. This rotation is described by [43],

$$
\mathcal{H}_{\mathrm{pol}}=\mathcal{D} \sum_{\substack{m, i \in \mathcal{C}_{m} \\ \alpha \neq \beta, \sigma}} \lambda_{\alpha \beta}\left(\mathbf{r}_{i}-\mathbf{R}_{m}\right)\left(\hat{d}_{i \alpha \sigma}^{\dagger} \hat{d}_{i \beta \sigma}+\hat{d}_{i \beta \sigma}^{\dagger} \hat{d}_{i \alpha \sigma}\right) .
$$

The orbital-polarization parameter $\mathcal{D}$ is defined by the matrix element $\left\langle i \alpha\left|v\left(\left|\mathbf{r}_{i}-\mathbf{R}_{m}\right|\right)\right| i \beta\right\rangle \equiv \mathcal{D} \lambda_{a b}\left(\mathbf{r}_{i}-\mathbf{R}_{m}\right)$. Here, we shall treat $\mathcal{D}$ as a free parameter. The sign of the matrix element is encoded in $\lambda_{\alpha \beta}\left(\mathbf{r}_{i}-\mathbf{R}_{m}\right)= \pm 1$ and depends on the vector $\mathbf{r}_{i}-\mathbf{R}_{m}$. For the $\{a, b\}$ doublet we have [42],

$$
\lambda_{a b}\left(\mathbf{r}_{i}-\mathbf{R}_{m}\right)=\left\{\begin{array}{ccc}
1 & \text { if } & \left(\mathbf{r}_{i}-\mathbf{R}_{m}\right) \|(111),(11 \overline{1}), \\
-1 & \text { if } & \left(\mathbf{r}_{i}-\mathbf{R}_{m}\right) \|(\overline{1} 11),(1 \overline{1} 1) .
\end{array}\right.
$$

Signs of all other $\lambda_{\alpha \beta}$ are obtained by cubic symmetry, see the Supplemental Material [54].

The effect of orbital polarization (2) on vanadium ions around a Ca defect is shown in Fig. 11(b) for the large $\mathcal{D}$ case. The actual form of the rotated $\left\{a^{\prime}, b^{\prime}, c^{\prime}\right\}$ orbitals depends on the corner of the defect cube under analysis. The orbitals are here classified according to their energy, see Fig. 11(c). This perturbation of the $G$-type $\mathrm{OO}$ is expected to be a strong effect as it involves the orbitals of all eight $\mathrm{V}$ ions in a defect cube. It competes with the CF, JT and the superexchange terms, which stabilize the $C-\mathrm{AF} / G$-AO order in $\mathrm{LaVO}_{3}$.

Each $\mathrm{Ca}^{2+}$ defect injects a hole that replaces the $b^{\prime}$ electron on a defect cube with the highest energy in the actual defect realization [31], see Fig. 1 (b). Which $\mathrm{V}$ ion this is depends on the interaction $v(r)$ with all other random defects and doped holes. The unrestricted Hartree-Fock (UHF) method is well designed to study spin-orbital order [55-58]. The subtle self-consistency problem, with random charged defects, is solved here using the rotationally invariant UHF method, which is able to reproduce the gap between the lower Hubbard band (LHB) and the UHB (with its multiplet structure) for the perovskite vanadates [31]. Statistical averages are performed over $M=100$ defect realizations, and we have

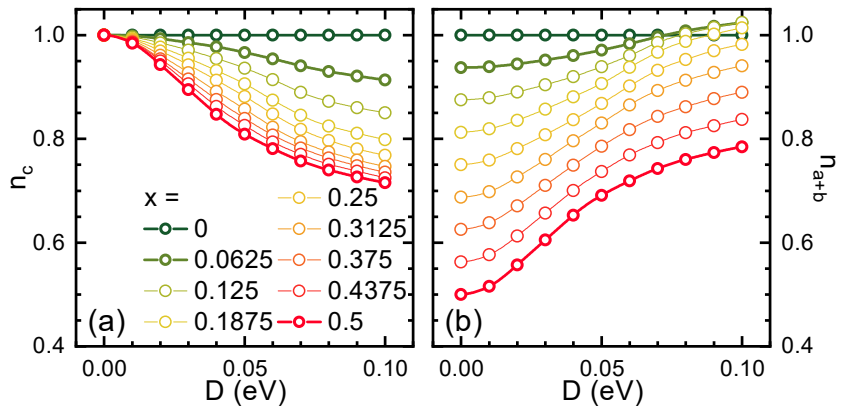

FIG. 2. Average electron density (per V ion) versus orbitalpolarization parameter $\mathcal{D}(2)$ for doping $x \in[0.0,0.5]$ [legend in (a)] for: (a) $c$ orbitals, $n_{c}$; (b) $\{a, b\}$ orbital doublet, $n_{a+b}$. Parameters: $U=4.5, J_{H}=0.5, t=0.2, V_{\mathrm{D}}=2.0($ all in $\mathrm{eV})$. 


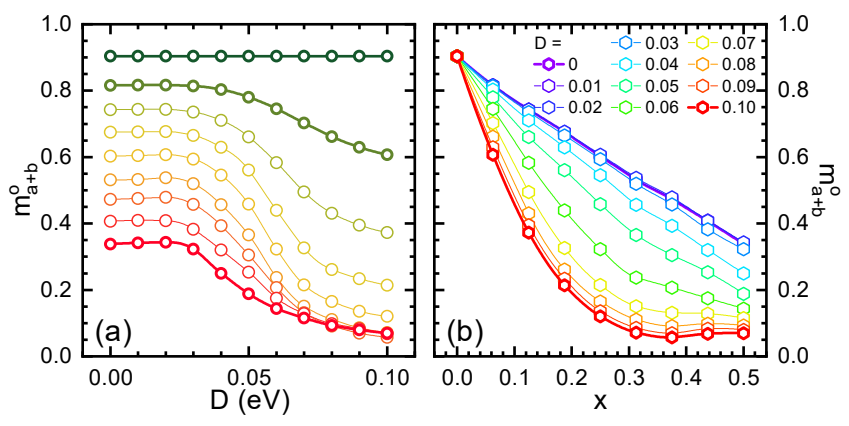

FIG. 3. Orbital order parameter $m_{a+b}^{o}$ (3): (a) for increasing orbital polarization $\mathcal{D}$ at different doping [same legend as in Fig. 22 a)] and (b) for increasing doping $x \in[0,0.5]$ at representative values of $\mathcal{D}$ (see legend). Parameters as in Fig. 2

verified that, for the quantities presented here, it suffices to consider $N=4 \times 4 \times 4$-size clusters.

In Fig. 2, we show how orbital polarization $\mathcal{D}$ influences charge densities $n_{c}$ and $n_{a+b} \equiv n_{a}+n_{b}$ for increasing doping $x$, where $n_{\alpha}=\left\langle\hat{n}_{\alpha}\right\rangle$ and $\hat{n}_{\alpha}=\frac{1}{N} \sum_{i \sigma} \hat{n}_{i \alpha \sigma}$. The case $\mathcal{D}=0$ is straightforward: doped holes go into the higher lying $a b$ states, i.e., $n_{a+b}=1-x$ and $n_{c}=1$. At finite $\mathcal{D}$, electrons occupy the rotated $\left|c^{\prime}\right\rangle$ and $\left|b^{\prime}\right\rangle$ orbitals that, for increasing $\mathcal{D}$, leads to a decrease of $n_{c}$ and to an increase of $n_{a+b}$, which may even exceed 1 . This redistribution is evident in the large $\mathcal{D}$ limit where the occupied states become $\left|c^{\prime}\right\rangle=(2|c\rangle-|a\rangle-|b\rangle) / \sqrt{6}$ and $\left|b^{\prime}\right\rangle=(|a\rangle-|b\rangle) / \sqrt{2}$ for a $\mathrm{V}$ ion in (111) position, see Fig. 1 (b), leading - for small $x$ and $t=0-$ to occupations $n_{a+b}=1-x+\frac{8}{3} x$ and $n_{c}=1-\frac{8}{3} x$.

The rotation of $t_{2 g}$ orbitals reduces the OO parameter describing the staggered $a / b$ order on each defect cube:

$$
m_{a+b}^{o} \equiv \frac{1}{M} \sum_{s=1}^{M} \frac{1}{N} \sum_{i}\left\langle\hat{n}_{i a}-\hat{n}_{i b}\right\rangle_{s} e^{i \mathbf{Q}_{G} \cdot \mathbf{R}_{i}},
$$

where $\mathbf{Q}_{G}=(\pi, \pi, \pi)$ is the vector corresponding to the $G$-AO order. One finds $m_{a+b}^{o} \simeq 0.9$ in the undoped case, see Fig. 3 (a), i.e., due to the finite hopping $t=0.2 \mathrm{eV}$. For $\mathcal{D}=0$, the order parameter $m_{a+b}^{o}$ decreases almost linearly with $x$. This case has been studied in a polaron theory using a small $t$ expansion [31] where $m_{a+b}^{o} \simeq 1-x\left(1+2 \delta_{c}\right)$. The $1-x$ describes the dilution of electrons in $a$ or $b$ orbitals upon doping. The polarity parameter $\delta_{c}$ is 0 if the doped hole is localized on a single $\mathrm{V}$ site, and is finite, but less than 0.5 , if it moves in a double exchange process along an active bond (AB) [43], thereby generating orbital defects. It is clear that the kinetic energy of holes in the $\mathcal{D}=0$ case [31] weakens the OO, but does not collapse it. In contrast, the $\mathcal{D}$ dependence in Fig. 3(a) is, for small $\mathcal{D} \leq 0.03 \mathrm{eV}$, almost absent and followed by a decay centered at $\mathcal{D}_{c} \approx 0.05$. We identify the orbital polarization interaction $\propto \mathcal{D}$ as the driving force of the decay. For large doping $x \geq x_{c} \approx 0.3$ and $\mathcal{D} \geq 0.07$ $\mathrm{eV}$, there is a saturation of $m_{a+b}^{o}$ induced by the large number of overlapping defect cubes. Simultaneously $C$-AF spin order persists in the regime where the $\mathrm{OO}$ melted. This behavior

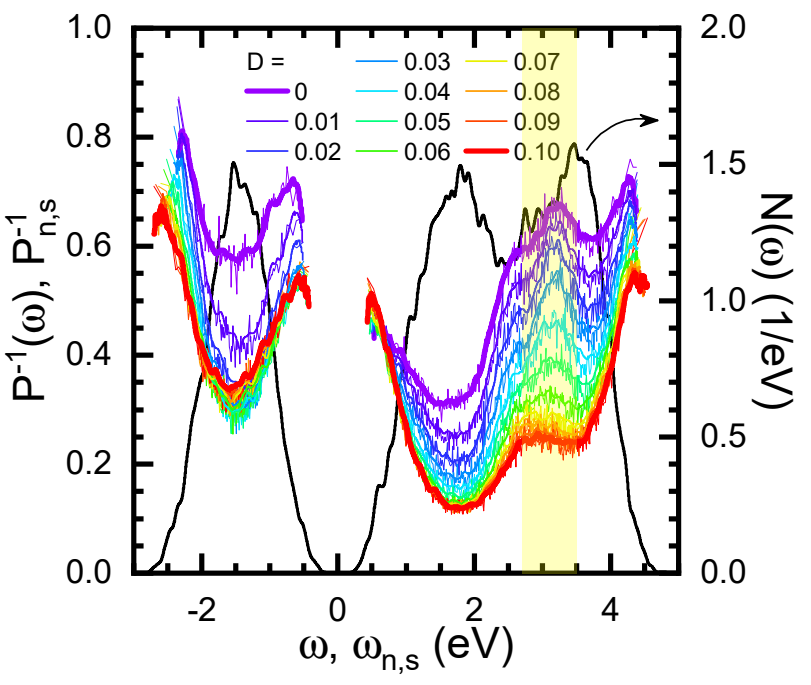

FIG. 4. IPN spectrum $P_{n, s}^{-1}$ versus $\omega_{n, s}$ and average $P^{-1}(\omega)$ for different $\mathcal{D}$ (see legend) at $x=0.3125$. Black lines show $N(\omega)$ with LHB/UHB for $\mathcal{D}=0$. The $\mathcal{D}$ dependence of HS $d^{2} \rightarrow d^{3}$ transitions on defect cubes at $\omega \in(2.7,3.5) \mathrm{eV}$, reflects the melting of $\mathrm{OO}$ (see shading). Parameters as in Fig. 2

agrees with experimental data [27--29].

We remark that the Hamiltonian parameters used here are relevant for $\mathrm{La}_{1-x} \mathrm{Sr}_{x} \mathrm{VO}_{3}$, where $G$-AO order disappears at $x_{\exp } \simeq 0.18$ [27]. It is worth noticing that the decay of the $\mathrm{OO}$ is due to a field term in the Hamiltonian, which explains its rather gradual decline, a trend also seen in experiments [28, 41]. So far, we have not observed in our data the collective features expected for conventional phase transitions.

Given the randomness of these systems, how does the localization of states change with orbital polarization $\propto \mathcal{D}$ ? A convenient measure of the degree of localization of a UHF wave function $\psi_{n, s}(r)$ is the participation number (PN) $P_{n, s}$, which is 1 for a state localized on a single site and $N$ for a Bloch state. Usually, one considers the inverse participation number (IPN) which takes the form [31], $P_{n, s}^{-1}=\sum_{i}\left(\sum_{\alpha \sigma}\left|\left\langle\psi_{n, s} \mid i \alpha \sigma\right\rangle\right|^{2}\right)^{2} \in[0,1]$ for systems with spin-orbital degeneracy. $P_{n, s}^{-1}$ is plotted in Fig. 4 for $x=0.3125$ versus the respective eigenvalues $\omega=\omega_{n, s}$ for all $6 N$ states $n$ and $M=100$ defect realizations $s$ together with the average IPN spectra $P(\omega)^{-1}$ [31]. Interestingly, despite the strong changes in the UHF wave functions $\psi_{n, s}(r)$, the density of states $N(\omega) \equiv \frac{1}{M} \sum_{s=1}^{M}\left[\frac{1}{N} \sum_{n=1}^{6 N} \delta\left(\omega-\omega_{n, s}\right)\right]$ hardly changes for $\mathcal{D} \leq 0.1 \mathrm{eV}$; thus we show it only for $\mathcal{D}=0$. Overall, one recognizes a gradual decrease of the IPN values with increasing $\mathcal{D}$ and a saturation for $\mathcal{D} \geq 0.08 \mathrm{eV}$, where the $\mathrm{OO}$ is practically absent. The PN results in maximum 3 (8) sites for the LHB (UHB): all states remain well localized. The discontinuity of the IPN at $\mathcal{D}=0$ between removal and addition states, right below and above the Fermi energy $\mu$, has been discussed before [31]. Here, we observe its disappearance at moderate $\mathcal{D}$ : delocalization of removal states can be attributed to the orbital rotation leading to $c^{\prime}$ or- 


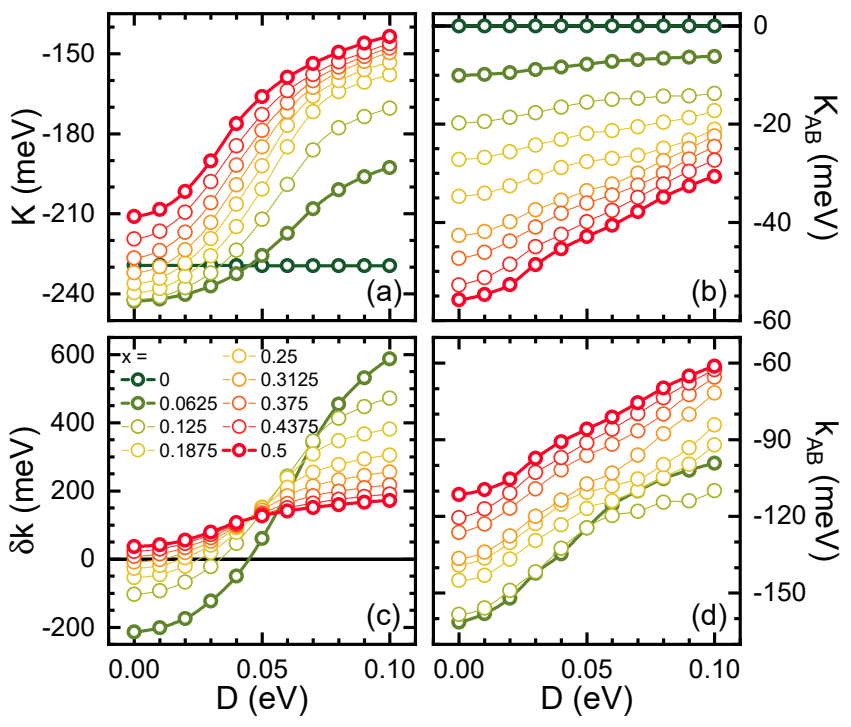

FIG. 5. Top - Kinetic energies per site versus orbital polarization strength $\mathcal{D}$ for different doping $x \in[0,0.5]$ [for color convention see legend in (c)]: (a) total energy $K$, and (b) the kinetic energy on active bonds $K_{\mathrm{AB}}$. Bottom - Energies per defect (4): (c) change of total kinetic energy $\delta k$ (relative to $x=0$ ), and (d) the kinetic energy on an active bond $k_{\mathrm{AB}}$. Parameters as in Fig. 2

\section{bital [59].}

For the $\mathcal{D}$ dependence of the IPN (Fig. 4), the energy interval $\omega \in(2.7,3.5) \mathrm{eV}$ is special and shows the largest variation in the range $0.04<\mathcal{D}<0.07 \mathrm{eV}$, similar to the $\mathcal{D}$ dependence of $m_{a+b}^{o}$ for $x=0.3125$, shown in Fig. 3(a). There are three different types of $d^{2} \rightarrow d^{3}$ transitions that fall into this energy window. Namely, either one of the two low spin (LS) transitions in the host or the high spin (HS) transition on a defect cube, where excitation energies are increased by $V_{D}$, i.e., $\omega_{\mathrm{HS}}=U-3 J_{H}+V_{D}+\omega_{\mathrm{LHB}} \approx 3.0 \mathrm{eV}$, where the position of the LHB is given by $\omega_{\mathrm{LHB}}=E_{\mathrm{LHB}}-\mu \approx-V_{D}$ [31]. It is the $\omega_{\mathrm{HS}}$ transitions that are sensitive to the melting of OO.

We have discussed above that the rotation of orbitals lowers the Coulomb energy of electrons in the electric field of defects. So far, we have not explained which mechanism opposes the rotation and determines the characteristic scales $\mathcal{D}_{c}$ and $x_{c}$ in Fig. 3. We show here that both are indeed determined by the kinetic energy of the system rather than by the $\mathrm{CF}$ - a consequence of strong correlations. First, we analyze in Fig. 5(a) the total kinetic energy per vanadium site, $K(x) \equiv \frac{1}{N}\left\langle\mathcal{H}_{\text {kin }}\right\rangle$, which includes both the hopping $\propto t$ and Fock $\propto v\left(r_{i j}\right)$ terms [43]. For the undoped Mott insulator $(x=0)$, such as $\mathrm{LaVO}_{3}$, we find large kinetic energy $K(0) \simeq-230 \mathrm{meV}$, see the horizontal $x=0$ line in Fig. 5.a). This is equivalent to the sum of the spin-orbital superexchange energies for the three cubic bond directions [60]. For all other $x \geq 0.0625$, one finds a monotonic increase of $K$ (i.e., loss of superexchange) for increasing either $x$ or $\mathcal{D}$. Note the complementary trends in the decay of the OO parameter $m_{a+b}^{o}$ in Fig. 3(a).
From a polaron perspective, the increase of $K$ is puzzling as one may expect that added holes would lead to delocalization, giving rise to some extra negative kinetic energy. In fact, for small $\mathcal{D}$ and $x$, the kinetic energy $K$ in Fig. 5(a) is indeed lower than the energy of the undoped system $K(0)$, in agreement with intuition. The dominant kinetic energy gain is expected to stem from $d^{2} d^{1} \rightarrow d^{1} d^{2}$ double exchange process on active FM bonds as confirmed by looking at the total UHF kinetic energy of holes on $\mathrm{ABs} K_{\mathrm{AB}}$, see Fig. 5(b). We also consider the kinetic energy gain per defect $\delta k$ and per active bond $k_{\mathrm{AB}}$, or equivalently per doped hole,

$$
\delta k \equiv[K(x)-K(0)] / x, \quad k_{\mathrm{AB}} \equiv K_{\mathrm{AB}}(x) / x,
$$

The kinetic energy gain $\delta k$ shown in Fig. 5(c) reveals an approximate isosbestic point, where $\delta k$ increases (decreases) as function of $x$ for small (large) $\mathcal{D}$. For $\mathcal{D}=0$ in the dilute case $(x=0.0625)$, the kinetic energy gain is $\delta k=-0.208$ $\mathrm{eV}$, while the kinetic energy of a hole on an active bond in Fig. 5 (d) is $k_{\mathrm{AB}} \approx-0.162 \mathrm{eV}$. To better appreciate these numbers we recall that $t=0.2 \mathrm{eV}$. Thus, we conclude that $k_{\mathrm{AB}}$ is in fact the dominant contribution of the total kinetic energy gain $\delta k$ at $x=0.0625$ and small $\mathcal{D}$. For larger doping and small $\mathcal{D}$, the kinetic energy per hole is quenched due to electronelectron and electron-defect interactions [31], and the formation of localized bipolarons (ABs with 2 doped holes) created by touching defect cubes [54].

Next, we turn to the $\mathcal{D}$ dependence of $\delta k$ and $k_{\mathrm{AB}}$ in Figs. 5 (c) and 5(d). For low (high) doping $x=0.0625$ (0.50), the change of $\delta k$ between $\mathcal{D}=0$ and $0.1 \mathrm{eV}$ is 800 (200) $\mathrm{meV}$, i.e., much more than the change of $k_{\mathrm{AB}}$ which is only 60 (40) meV. This clearly shows that the $\mathcal{D}$-dependent change of $\delta k$ is mainly due to the orbital rotation at all corners of the defect cube and not just at the active bond. The smaller values at high doping result from the frustration of orbital rotation due to the touching of defect cubes. In view of the significant overlap of defect cubes at already moderate doping, one may expect that some states extend over several cubes. Yet, in the analysis of IPN we have shown in Fig. 4 that such delocalized states do not exist and holes injected into the LHB do extend typically just over two to three $\mathrm{V}$ sites.

Summarizing, we have shown that the dominant mechanism that leads to the collapse of the orbital order is not the motion of doped holes, but the orbital rotation induced by charged defects on their vanadium neighbors. This field induced suppression of the orbital order is non-cooperative and does not lead to a conventional phase transition, - like the loss of antiferromagnetic order in high- $T_{c}$ cuprates [61]. We believe that our model gives a qualitative explanation of the decay of the orbital order accompanied by robustness of spin order in $R_{1-x} \mathrm{Sr}_{x} \mathrm{VO}_{3}$ compounds.

A. M. O. acknowledges Narodowe Centrum Nauki (NCN, Poland) Project No. 2016/23/B/ST3/00839 and is grateful for the Alexander von Humboldt Foundation Fellowship (Humboldt-Forschungspreis). 


\section{Appendix: Supplemental Material}

In the first Section of this Supplemental Material, we present the three-band extended Hubbard model used in the main text, which describes the $t_{2 g}$ electronic states in vanadium perovskites. In Section II, we explain in detail how the general form of the orbital polarization interaction, describing the rotation of $t_{2 g}$ orbitals on the vanadium neighbors of charged defects, is obtained from the representative term acting on the $\{a, b\}$ orbital doublet reported in the main text. In Section III, we show that spin order decouples from orbital order and is robust at increasing doping; it is not influenced by orbital polarization interaction. Finally, in Section IV, we discuss some of the interaction and frustration effects appearing on increasing defect density where more and more defect cubes have common corners, edges or faces.

\section{The three-band Hubbard model}

The Hamiltonian for $t_{2 g}$ electrons in doped vanadium $(\mathrm{La}, \mathrm{Y})_{1-x} \mathrm{Ca}_{x} \mathrm{VO}_{3}$ perovskites [42, 43],

$$
\mathcal{H}_{t 2 g}=\mathcal{H}_{\mathrm{Hub}}+\mathcal{H}_{\mathrm{pol}}+\sum_{i<j} v\left(r_{i j}\right) \hat{n}_{i} \hat{n}_{j}+\sum_{m i} v\left(r_{m i}\right) \hat{n}_{i},
$$

includes the three-band Hubbard model $\mathcal{H}_{\mathrm{Hub}}$ [44] for the reference host system without charged defects. It acts on the electrons in $t_{2 g}$ orbital states similar to the model for pnictides [45] and consists of the kinetic energy $\mathcal{H}_{\text {kin }}$, local interactions described by the degenerate Hubbard model $\mathcal{H}_{\mathrm{U}-\mathrm{J}_{\mathrm{H}}}$, supplemented by rather weak terms: the $\mathrm{CF}$ splitting $\mathcal{H}_{\mathrm{CF}}$, and the JT interactions $\mathcal{H}_{\mathrm{JT}}$,

$$
\mathcal{H}_{\mathrm{Hub}}=\mathcal{H}_{\text {kin }}+\mathcal{H}_{\mathrm{U}-\mathrm{J}_{\mathrm{H}}}+\mathcal{H}_{\mathrm{CF}}+\mathcal{H}_{\mathrm{JT}} .
$$

The kinetic energy reads as,

$$
\mathcal{H}_{\text {kin }}=\sum_{\substack{\langle i j\rangle \| \gamma \\ \alpha \sigma \sigma}} t_{i j}^{\gamma \alpha}\left(\hat{d}_{i \alpha \sigma}^{\dagger} \hat{d}_{j \alpha \sigma}+\hat{d}_{j \alpha \sigma}^{\dagger} \hat{d}_{i \alpha \sigma}\right) .
$$

Here, $\hat{d}_{i \alpha \sigma}^{\dagger}$ is the electron creation operator in the $t_{2 g}$ orbitals $\alpha \in\{x y, y z, z x\}$ with spin $\sigma=\uparrow, \downarrow$ at site $i$. The effective hopping $t_{i j}^{\gamma \alpha}$ of $t_{2 g}$ electrons between two vanadium ions at sites $i$ and $j$ depends on bond direction $\langle i j\rangle \| \gamma$ and on the orbital flavor $\alpha$. It occurs via hybridization with an intermediate oxygen $2 p_{\pi}$ orbital along $180^{\circ} \mathrm{V}-\mathrm{O}-\mathrm{V}$ bonds. Therefore, the hopping: (i) is diagonal and conserves the orbital flavor $\alpha$ when $\alpha \neq \gamma$ and hybridization is finite, i.e., $t_{i j}^{\gamma \alpha}=-t$, and (ii) vanishes in one of the three cubic directions for which the hybridization with oxygen $2 p_{\pi}$ orbitals vanishes by symmetry, i.e., $t_{i j}^{\gamma \gamma}=0$. Using these properties, it is convenient to introduce the following short-hand notation for the orbital degree of freedom [51],

$$
|a\rangle \equiv|y z\rangle, \quad|b\rangle \equiv|z x\rangle, \quad|c\rangle \equiv|x y\rangle,
$$

with the labels $\gamma=a, b, c$ referring to the cubic axis along which the hopping element vanishes.

Local interactions at vanadium ions are described by the degenerate Hubbard model $\mathcal{H}_{\mathrm{U}-\mathrm{J}_{\mathrm{H}}}$ parametrized by two Kanamori parameters: intraorbital Coulomb interaction $U$ and Hund's exchange $J_{H}$ between two $t_{2 g}$ electrons [52],

$$
\begin{aligned}
\mathcal{H}_{\mathrm{U}-J_{\mathrm{H}}} & =U \sum_{i \alpha} \hat{n}_{i \alpha \uparrow} \hat{n}_{i \alpha \downarrow}+J_{H} \sum_{i, \alpha \neq \beta} \hat{d}_{i \alpha \uparrow}^{\dagger} \hat{d}_{i \alpha \downarrow}^{\dagger} \hat{d}_{i \beta \downarrow} \hat{d}_{i \beta \uparrow} \\
& +\sum_{i, \alpha<\beta}\left[\left(U-\frac{5}{2} J_{H}\right) \hat{n}_{i \alpha} \hat{n}_{i \beta}-2 J_{H} \hat{\overrightarrow{\vec{S}}}_{i \alpha} \cdot \hat{\vec{S}}_{i \beta}\right] .
\end{aligned}
$$

Interorbital Coulomb interactions $\propto n_{i \alpha} n_{i \beta}$ are expressed in terms of spin-orbital electron density operators, $\hat{n}_{i \alpha}=\sum_{\sigma} \hat{n}_{i \alpha \sigma}=\sum_{\sigma} \hat{d}_{i \alpha \sigma}^{\dagger} \hat{d}_{i \alpha \sigma}$; orbital spin operators, $\hat{\vec{S}}_{i \alpha} \equiv\left\{\hat{S}_{i \alpha}^{x}, \hat{S}_{i \alpha}^{y}, \hat{S}_{i \alpha}^{z}\right\}$, appear in the Hund's exchange $\propto-J_{H} \hat{\vec{S}}_{i \alpha} \cdot \hat{\vec{S}}_{i \beta}$. In a Mott insulator, charge fluctuations are quenched and electrons localize due to large $U \gg t$. In case of $\mathrm{LaVO}_{3}$, one finds a $t_{2 g}^{2}$ configuration at each vanadium ion and Hund's exchange $J_{H}$ stabilizes high spin states with $S=1$. The insulating ground state of $\mathrm{LaVO}_{3}$ has a $C$-type antiferromagnetic $(C-\mathrm{AF})$ spin coexisting with $G$-type alternating orbital $(G-\mathrm{AO})$ order [44].

The structural transition at $T_{s} \sim 200 \mathrm{~K}$ lifts the degeneracy of the three $t_{2 g}$ orbitals and breaks the cubic symmetry in the orbital space [44]. At low temperature, the CF splitting favors $x y \equiv c$ orbitals by energy $\Delta_{c}=0.1 \mathrm{eV}$, which we take as a constant parameter independent of temperature, and the $\mathrm{CF}$ Hamiltonian is,

$$
\mathcal{H}_{\mathrm{CF}}=-\Delta_{c} \sum_{i} \hat{n}_{i c} .
$$

It selects the orbital doublet as orbital degree of freedom and gives either $c_{i}^{1} a_{i}^{1}$ or $c_{i}^{1} b_{i}^{1}$ configuration at the $\mathrm{V}$ ion sitting at site $i$, depending on the actual lattice distortion in the $a b$ plane. In a Mott insulator, spin-orbital superexchange explains the ground state observed in $\mathrm{LaVO}_{3}$ [21].

Lattice distortions change the electronic state and induce weak JT interactions in the three-band model (A.2),

$$
\begin{aligned}
\mathcal{H}_{\mathrm{JT}} & =\frac{1}{4} V_{a b} \sum_{\langle i j\rangle \| a b}\left(\hat{n}_{i a}-\hat{n}_{i b}\right)\left(\hat{n}_{j a}-\hat{n}_{j b}\right) \\
& -\frac{1}{4} V_{c} \sum_{\langle i j\rangle \| c}\left(\hat{n}_{i a}-\hat{n}_{i b}\right)\left(\hat{n}_{j a}-\hat{n}_{j b}\right) .
\end{aligned}
$$

Using the orbital $\tau_{i}^{z}$ operators,

$$
\tau_{i}^{z} \equiv \frac{1}{2} \sum_{\sigma}\left(\hat{d}_{i a \sigma}^{\dagger} \hat{d}_{i a \sigma}-\hat{d}_{i b \sigma}^{\dagger} \hat{d}_{i b \sigma}\right),
$$

the JT interactions are,

$$
\mathcal{H}_{\mathrm{JT}}=V_{a b} \sum_{\langle i j\rangle \| a b} \hat{\tau}_{i}^{z} \hat{\tau}_{j}^{z}-V_{c} \sum_{\langle i j\rangle \| c} \hat{\tau}_{i}^{z} \hat{\tau}_{j}^{z} .
$$


These interactions stabilize another competing type of spinorbital order [21], the $G$-type $\mathrm{AF}$ ( $G$-AF) spin coexisting with $C$-type $\mathrm{AO}(C$-AO) order, which represents the ground state in $\mathrm{YVO}_{3}$ [32, 42]. Small doping $x \simeq 0.01$ leads to a phase transition to the $C-\mathrm{AF} / G-\mathrm{AO}$ phase, which is the phase studied in this work.

Following the earlier studies, we have fixed the small parameters in $\mathcal{H}_{\mathrm{CF}}$ and $\mathcal{H}_{\mathrm{JT}}$ as follows: $\Delta_{c}=0.1, V_{a b}=0.03$, and $V_{c}=0.05$ (all in $\mathrm{eV}$ ). The term $\propto V_{a b}$ favors alternating $\{a, b\}$ orbitals, i.e., $\mathrm{AO}$ order in the $a b$ planes $\left(V_{a b}>0\right)$ while the ferro-orbital order is favored along the $c$ cubic axis $\left(V_{c}>0\right)$. Thus, the term $\propto V_{c}$ weakens the superexchange orbital interaction $\propto J r_{1}$, where $J=4 t^{2} / U$ and $r_{1}=(1-3 \eta)^{-1}$ with $\eta=J_{H} / U$, which along the $c$ axis favors the observed $G$-AO order [21]. One finds that for the present parameters ( $U=4.5, t=0.2, J_{H}=0.5$, all in eV) $J r_{1}=53 \mathrm{meV}$, so taking $V_{c}=50 \mathrm{meV}$ one is indeed close to the switching of the orbital order observed in $\mathrm{YVO}_{3}[35,62]$.

\section{Orbital polarization around defects}

The orbital polarization term results from electron-defect interaction and modifies the orbital basis at $\mathrm{V}$ ions on the $d e$ fect cube $\mathcal{C}_{m}$ around the charged defect at $\mathbf{R}_{m}$ [43]:

$$
\mathcal{H}_{\mathrm{pol}}=\mathcal{D} \sum_{\substack{m, i \in \mathcal{C}_{m} \\ \alpha \neq \beta, \sigma}} \lambda_{\alpha \beta}\left(\mathbf{r}_{i}-\mathbf{R}_{m}\right)\left(\hat{d}_{i \alpha \sigma}^{\dagger} \hat{d}_{i \beta \sigma}+\hat{d}_{i \beta \sigma}^{\dagger} \hat{d}_{i \alpha \sigma}\right) .
$$

The coefficients $\lambda_{\alpha \beta}\left(\mathbf{r}_{i}-\mathbf{R}_{m}\right)= \pm 1$ are selected to minimize the Coulomb repulsion with the defect charge. Taking the $\{a, b\}$ doublet active along the $c$ axis as an example, one finds that $\lambda_{a b}\left(\mathbf{r}_{i}-\mathbf{R}_{m}\right)=+1$ for the directions $\left(\mathbf{r}_{i}-\mathbf{R}_{m}\right) \|(111)$, $\left(\mathbf{r}_{i}-\mathbf{R}_{m}\right) \|(11 \overline{1})$, and -1 for the other two diagonal directions [42], see Table I. For this doublet the eigenstates of the polarization operator,

$$
\tau_{i}^{x} \equiv \frac{1}{2} \sum_{\sigma}\left(\hat{d}_{i a \sigma}^{\dagger} \hat{d}_{i b \sigma}+\hat{d}_{i b \sigma}^{\dagger} \hat{d}_{i a \sigma}\right)
$$

at site $i$ have energy either lowered or increased by $\mathcal{D}$, depending on whether they are directed towards the defect site $m$ or have lobes in the plane being orthogonal to the above direction, see Fig. 1 (main text).

The remaining values of $\lambda_{\alpha \beta}\left(\mathbf{r}_{i}-\mathbf{R}_{m}\right)$, which determine the local mixing of $\{a, c\}$ or $\{b, c\}$ orbitals in analogy to Eq.

TABLE I. The coefficients $\lambda_{\alpha \beta}\left(\mathbf{r}_{i}-\mathbf{R}_{m}\right)$ in Eq. 22 for different orbital doublets $\{\alpha, \beta\}$ and for different directions $\left\{\left(\mathbf{r}_{i}-\mathbf{R}_{m}\right)\right\}$.

\begin{tabular}{ccccc}
\hline \hline orbital doublet & \multicolumn{4}{c}{$\left(\mathbf{r}_{i}-\mathbf{R}_{m}\right) \|$} \\
$\{\alpha, \beta\}$ & $(111)$ & $(11 \overline{1})$ & $(\overline{1} 11)$ & $(1 \overline{1} 1)$ \\
\hline$\{a, b\}$ & 1 & 1 & -1 & -1 \\
$\{a, c\}$ & 1 & -1 & -1 & 1 \\
$\{b, c\}$ & 1 & -1 & 1 & -1 \\
\hline \hline
\end{tabular}

A.10, may be obtained by simultaneous cyclic permutations of the orbitals $\{a, b, c\}$ and of the cubic axes in the direction of the vector $\left(\mathbf{r}_{i}-\mathbf{R}_{m}\right)$, see Table I. Note that each direction along one of the cube's diagonals involves two vanadium ions.

\section{Orbital polarization dependence of spin order}

The defect-induced orbital polarization (2) strongly affects the orbital order as well as the doping dependence of the $G$ type orbital order parameter $m_{a b}^{o}$, as we have shown in Fig. 3(b). Here, we present a complementary picture that shows how the decrease of spin order parameter, $m^{s}$, in the $C$-AF phase as a function of doping $x$, changes with the orbital polarization strength $\mathcal{D}$. The spin order parameter is defined as,

$$
m^{s}=\sum_{i, \nu}\left\langle\hat{d}_{i \nu \uparrow}^{\dagger} \hat{d}_{i \nu \uparrow}-\hat{d}_{i \nu \downarrow}^{\dagger} \hat{d}_{i \nu \downarrow}\right\rangle e^{i \mathbf{Q}_{C} \cdot \mathbf{r}_{i}}
$$

where $\mathbf{Q}_{C} \equiv(\pi, \pi, 0)$, and the sum is over all sites $\mathbf{r}_{i}$ and orbital flavors $\nu=a, b, c$.

The results in Fig. 6 6 show that the spin order parameter $m^{s}$ has an approximate linear decay with $x$, and only an extremely weak dependence on $\mathcal{D}$. The latter may be easily understood by the argument that orbital rotation does not affect spins. Yet, a so weak dependence on $\mathcal{D}$ is surprising if we go back to the origins of the decay with $x$. The latter has been explained in terms of a spin-polaron approach in Ref. [31]. There are two basic contributions to the almost linear decrease of $m^{s}$ with $x$ :

(a) the dilution of spins due to the added holes and

(b) the kinetic energy or string-formation effect due to the motion of doped holes in the $C$-AF background.

Orbital rotation leads to the mixing of flavors and thus to the

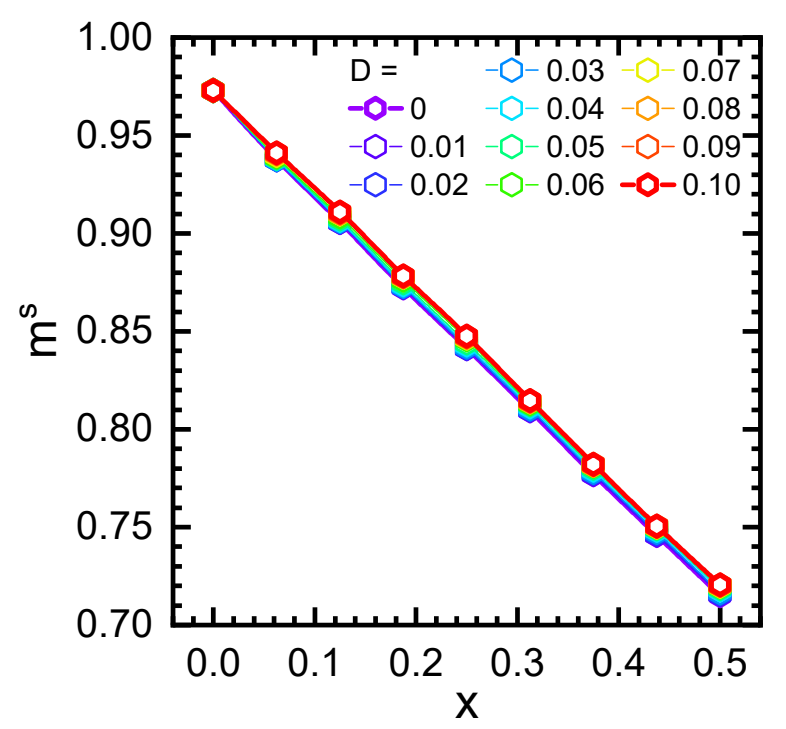

FIG. 6. Spin-order parameter $m^{s}$ versus doping $x$ and its dependence on the orbital polarization strength $\mathcal{D}$. Parameters as in the main text. 
appearance of off-diagonal hopping processes that affect the kinetic energy. From this perspective, the insensitivity of the spin-order to orbital rotation, which we observe in Fig. 3 , comes as a surprise. The solution of the puzzle follows from the observation that holes form small spin-orbital polarons that are bound to defects where the kinetic energy string contributions are small.

We note that similar trends were reported in a recent experimental study of spin and orbital disordering by hole doping in $\operatorname{Pr}_{1-x} \mathrm{Ca}_{x} \mathrm{VO}_{3}$ [41]. In that system, the long-range $C$-type $(C$-AF) spin order persists beyond the insulator-metal transition crossover regime $(0.22<x<0.25)$ and a Néel transition is still observed in the regime where the orbital order melted.

\section{Frustration resulting from overlapping defect cubes}

As we have argued in the text, the self-consistent UHF algorithm is capable to obtain the electronic structure of the doped Mott insulator even at high doping, where defect cubes share faces, edges or just corners. The final results presented in the paper are averages over many defect realizations - nevertheless one certainly would like to get some deeper insight or idea of the energy changes resulting from orbital rotations beyond the dilute limit, that is when frustration due to overlapping defect cubes is essential. This is possible by a careful analysis of correlation functions of individual random systems. In the following, we give for the interested reader a qualitative description of the most important effects due to the touching of defect cubes.

The increase with doping $x$ of the number of defect cubes sharing corners, edges, and faces is responsible for the nonlinearity with doping $x$ of the behavior of $n_{c}, n_{a+b}$ and $m_{a+b}^{o}$ at finite $\mathcal{D}$. Actually, the percolation limit for defect cubes taking into account simultaneously corner, edge and face sharing between $\mathrm{V}$ cubes is counterintuitively low: $x_{p}=0.0976$ [63]. This explains why $x=0.0625$ is somehow different from all other dopings we report. This also says that having defect cubes that share, in particular, one vertical bond is not that unusual also for very low values of doping. This brings in the possibility to confine two holes on that bond and gain substantial Coulomb potential energy (minimizing simultaneously the distances between the two holes and the two defects) at the expense of the kinetic energy gain we usually have at $a c$ tive bonds. In other words, small spin-orbital polarons merge to give bipolarons. This becomes more and more relevant for increasing values of $\mathcal{D}$ as active bonds gain less and less kinetic energy because of the induced orbital rotation.

It is worth noting that two defect cubes sharing a face along $a$ or $b$ direction can simultaneously gain Coulomb potential energy and kinetic energy by confining the two holes over the two shared vertical bonds, but this leads again to two active bonds as if they would be on separate defect cubes [63]. Also sharing just corners, horizontal edges and faces along the $c$ direction does not change the actual number of active bonds and has effects only on the potential energy one can gain. Accord- ingly, only defect cubes sharing vertical bonds really affects the kinetic energy of the system as they can reduce the overall number of active bonds.

Actually, the main source of kinetic energy loss with increasing $x$ is just such sharing of empty vertical bonds (occupied by a bipolaron). The loss of kinetic energy by orbital rotation on all vertical bonds of a defect cube on increasing $\mathcal{D}$ is the other relevant source of kinetic energy loss. In the dilute limit $(x=0.0625)$ for large enough $\mathcal{D}$, we have that defect realizations with shared vertical bonds become so much more favorable and, therefore, so much more easy to converge numerically that they dominate the statistical averages. Just for this doping, at $\sim \mathcal{D}_{c}$, one has a transition from a situation with mainly well separated defect cubes to mainly couples of defect cubes sharing one vertical bond, see Fig. 5(d). As a matter of fact, $\mathcal{D}$ affects also the way polarons interact: below $\mathcal{D}_{c}$, they avoid each other to maximize the gain in kinetic energy, but above $\mathcal{D}_{c}$, they attract each other in order to minimize the loss in kinetic energy.

Indeed, $\mathcal{D}_{c}$ corresponds to the value of $\mathcal{D}$ that imposes an orbital rotation large enough to make the kinetic energy gain on an active bond equal to just half of that of a standard superexchange bond, making thus equal the kinetic energy gain on two separated defect cubes (two active bonds and six spectator bonds [31]) and two defect cubes sharing an empty vertical bond: no active bonds, with no kinetic energy gain at all on the empty shared vertical bond, still six spectator bonds, but a whole ordinary superexchange bond recovered! Such an occurrence makes clear why at $\mathcal{D}_{c}$ the kinetic energy per defect is almost completely independent of $x$ (we have an approximate isosbestic point). The value of $x$ just rules the number of defect cubes sharing an empty vertical bond and if the energy of the two relevant configurations (two active bonds or one empty vertical bond plus a recovered ordinary superexchange bond) is equal, the dependence on $x$ is clearly lost. The small loss of kinetic energy $\delta k$ at $\mathcal{D}_{c}$, is then equal to that of a missing ordinary superexchange bond plus the difference between the kinetic energy of six ordinary superexchange bonds and the kinetic energy of six rotated spectator bonds.

It is now clear that the presence of defect cubes sharing empty vertical bonds (forming bipolarons) is very relevant for larger and larger values of doping this comes to dominate the physical properties. This is extremely clear by looking at $m_{a+b}^{o}$ as a function of $x$ for all finite values of $\mathcal{D}$. On increasing $x>x_{c} \approx 0.3$, one adds defects in $\mathrm{V}$ cubes that have almost all corners/edges already belonging to other defect cubes [63]. Hence, no substantial increase of rotations and decrease of orbital order above a certain value of $\mathcal{D}=0.07>\mathcal{D}_{c}$. Each added hole either generates a polaron (on an active bond) or a bipolaron (on an empty vertical bond). The latter actually slightly increases the orbital order, as it is clearly shown by $m_{a+b}^{o}$ versus $x$ in the regime of large values of $\mathcal{D}$. The position of the minimum defines $x_{c}$ where the balance is reached between decreasing the orbital order through the the formation of new spectator bonds and its increase by changing polarons into bipolarons. It also coincides with value at which $K$, as a 
function of $x$ for $\mathcal{D}=0$, passes through the undoped value. The gain of kinetic energy at polarons (active bonds) is again balanced by its loss at bipolarons (empty bonds).

Just one final remark regarding the role of $\mathcal{D}_{c}$ in the IPN: above $\mathcal{D}_{c}$, in the middle of the LHB, and less evidently right below $\mu$, the delocalization inverts its overall trend and actually decreases as the orbital polarization inhibits the gain of kinetic energy along the active bonds. Right above $\mu$, this mechanism works for all values of $\mathcal{D}$.

[1] B. Keimer, S. A. Kivelson, M. R. Norman, S. Uchida, J. Zaanen, Nature 518, 179 (2015).

[2] M. Imada, A. Fujimori, and Y. Tokura, Rev. Mod. Phys. 70, 1039 (1998).

[3] F. Grusdt, M. Kánasz-Nagy, A. Bohrdt, C. S. Chiu, G. Ji, M. Greiner, D. Greif, and E. Demler, Phys. Rev. X 8, 011046 (2018); A. Bohrdt, D. Greif, E. Demler, M. Knap, and F. Grusdt, Phys. Rev. B 97, 125117 (2018).

[4] Y. Zhou, K. Kanoda, and T.-K. Ng, Rev. Mod. Phys. 89, 025003 (2017).

[5] S. Uchida, T. Ido, H. Takagi, T. Arima, Y. Tokura, and S. Tajima, Phys. Rev. B 43, 7942 (1991); B. Keimer, N. Belk, R. J. Birgeneau, A. Cassanho, C. Y. Chen, M. Greven, M. A. Kastner, A. Aharony, Y. Endoh, R. W. Erwin, and G. Shirane, ibid. 46, 14034 (1992).

[6] M. A. Kastner, R. J. Birgeneau, G. Shirane, and Y. Endoh, Rev. Mod. Phys. 70, 897 (1998).

[7] P. A. Lee, N. Nagaosa, and X.-G. Wen, Rev. Mod. Phys. 78, 17 (2006).

[8] D. J. Scalapino, Rev. Mod. Phys. 84, 1383 (2012).

[9] G. Ghiringhelli, M. Le Tacon, M. Minola, S. Blanco-Canosa, C. Mazzoli, N. B. Brookes, G. M. De Luca, A. Frano, D. G. Hawthorn, F. He, T. Loew, N. Moretti Sala, D. C. Peets, M. Salluzzo, E. Schierle, R. Sutarto, G. A. Sawatzky, E. Weschke, B. Keimer, and L. Braicovich, Science 337, 6096 (2012).

[10] E. Fradkin, S. A. Kivelson, and J. M. Tranquada, Rev. Mod. Phys. 87, 457 (2015).

[11] Y. Tokura, Rep. Prog. Phys. 69, 797 (2006).

[12] E. Dagotto, T. Hotta, and A. Moreo, Phys. Rep. 3441 (2001).

[13] M. Quijada, J. Černe, J. R. Simpson, H. D. Drew, K. H. Ahn, A. J. Millis, R. Shreekala, R. Ramesh, M. Rajeswari, and T. Venkatesan, Phys. Rev. B 58, 16093 (1998).

[14] R. Kilian and G. Khaliullin, Phys. Rev. B 60, 13458 (1999).

[15] G. Giovannetti, S. Kumar, J. van den Brink, and S. Picozzi, Phys. Rev. Lett. 103, 037601 (2009).

[16] S. Yunoki, A. Moreo, E. Dagotto, S. Okamoto, S. S. Kancharla, and A. Fujimori, Phys. Rev. B 76, 064532 (2007).

[17] A. Charnukha, A. Cvitkovic, T. Prokscha, D. Pröpper, N. Ocelic, A. Suter, Z. Salman, E. Morenzoni, J. Deisenhofer, V. Tsurkan, A. Loidl, B. Keimer, and A. V. Boris, Phys. Rev. Lett. 109, 017003 (2012).

[18] Y. W. Cao, X. R. Liu, M. Kareev, D. Choudhury, S. Middey, D. Meyers, J. W. Kim, P. J. Ryan, J. W. Freeland, and J. Chakhalian, Nature Comm. 7, 10418 (2016).

[19] L. Kuerten, C. Richter, N. Mohanta, T. Kopp, A. Kampf, J. Mannhart, and H. Boschker, Phys. Rev. B 96, 014513 (2017).

[20] H. Lei, W.-G. Yin, Z. Zhong, and H. Hosono, Phys. Rev. B 89, 020409(R) (2014).

[21] G. Khaliullin, P. Horsch, and A. M. Oleś, Phys. Rev. Lett. 86,
3879 (2001); Phys. Rev. B 70, 195103 (2004).

[22] G. Khaliullin, Prog. Theor. Phys. Suppl. 160, 155 (2005).

[23] M. De Raychaudhury, E. Pavarini, and O. K. Andersen, Phys. Rev. Lett. 99, 126402 (2007).

[24] J.-Q. Yan, J.-S. Zhou, and J. B. Goodenough, Phys. Rev. Lett. 93, 235901 (2004); J.-S. Zhou, J. B. Goodenough, J.-Q. Yan, and Y. Ren, ibid. 99, 156401 (2007); J.-Q. Yan, J.-S. Zhou, J. B. Goodenough, Y. Ren, J. G. Cheng, S. Chang, J. Zarestky, O. Garlea, A. Llobet, H. D. Zhou, Y. Sui, W. H. Su, and R. J. McQueeney, ibid. 99, 197201 (2007).

[25] J. Reul, A. A. Nugroho, T. T. M. Palstra, and M. Grüninger, Phys. Rev. B 86, 125128 (2012).

[26] M. Kasuya, Y. Tokura, T. Arima, H. Eisaki, and S. Uchida, Phys. Rev. B 47, 6197 (1993).

[27] S. Miyasaka, T. Okuda, and Y. Tokura, Phys. Rev. Lett. 85, 5388 (2000).

[28] J. Fujioka, S. Miyasaka, and Y. Tokura, Pnys. Rev. B 72, 024460 (2005).

[29] J. Fujioka, S. Miyasaka, and Y. Tokura, Phys. Rev. Lett. 97, 196401 (2006); Phys. Rev. B 77, 144402 (2008).

[30] A. Avella, A. M. Oleś, and P. Horsch, Phys. Rev. Lett. 115, 206403 (2015).

[31] A. Avella, A. M. Oleś, and P. Horsch, Phys. Rev. B 97, 155104 (2018).

[32] Y. Ren, T. T. M. Palstra, D. I. Khomskii, A. A. Nugroho, A. A. Menovsky, and G. A. Sawatzky, Phys. Rev. B 62, 6577 (2000).

[33] S. Miyasaka, Y. Okimoto, M. Iwama, and Y. Tokura, Phys. Rev. B 68, 100406(R) (2003); S. Miyasaka, J. Fujioka, M. Iwama, Y. Okimoto, and Y. Tokura, ibid. 73, 224436 (2006).

[34] I. V. Solovyev, Phys. Rev. B 74, 054412 (2006).

[35] J. Fujioka, T. Yasue, S. Miyasaka, Y. Yamasaki, T. Arima, H. Sagayama, T. Inami, K. Ishii, and Y. Tokura, Pnys. Rev. B 82, 144425 (2010).

[36] J.-Q. Yan, J.-S. Zhou, J. G. Cheng, J. B. Goodenough, Y. Ren, A. Llobet, and R. J. McQueeney, Phys. Rev. B 84, 214405 (2011).

[37] P. Horsch, A. M. Oleś, L.F. Feiner, and G. Khaliullin, Phys. Rev. Lett. 100, 167205 (2008).

[38] M. Noguchi, A. Nakazawa, S. Oka, T. Arima, Y. Wakabayashi, H. Nakao, and Y. Murakami, Phys. Rev. B 62, R9271 (2000).

[39] G. R. Blake, T. T. M. Palstra, Y. Ren, A. A. Nugroho, and A. A. Menovsky, Phys. Rev. Lett. 87, 245501 (2001); Phys. Rev. B 65, 174112 (2002).

[40] M. H. Sage, G. R. Blake, and T. T. M. Palstra, Phys. Rev. B 77, 155121 (2008).

[41] M. Reehuis, C. Ulrich, P. M. Abdala, P. Pattison, G. Khaliullin, J. Fujioka, S. Miyasaka, Y. Tokura, and B. Keimer, Phys. Rev. B 94, 104436 (2016).

[42] P. Horsch and A. M. Oleś, Phys. Rev. B 84, 064429 (2011).

[43] A. Avella, P. Horsch, and A. M. Oleś, Phys. Rev. B 87, 045132 (2013).

[44] A. M. Oleś, P. Horsch, and G. Khaliullin, Phys. Rev. B 75, 184430 (2007).

[45] M. Daghofer, A. Nicholson, A. Moreo, and E. Dagotto, Phys. Rev. B 81, 014511 (2010); A. Georges, L. d'Medici, and J. Mravlje, Annu. Rev. Condens. Matter Phys. 4, 137 (2013); K. M. Stadler, Z. P. Yin, J. von Delft, G. Kotliar, and A. Weichselbaum, Phys. Rev. Lett. 115, 136401 (2015).

[46] S. Ishihara, Phys. Rev. Lett. 94, 156408 (2005).

[47] M. Daghofer, K. Wohlfeld, A. M. Oleś, E. Arrigoni, and P. Horsch, Phys. Rev. Lett. 100, 066403 (2008); P. Wróbel and A. M. Oleś, ibid. 104, 206401 (2010).

[48] V. Bisogni, K. Wohlfeld, S. Nishimoto, C. Monney, Jan Trinck- 
auf, K. Zhou, R. Kraus, K. Koepernik, C. Sekar, V. Strocov, B. Büchner, T. Schmitt, J. van den Brink, and J. Geck, Phys. Rev. Lett. 114, 096402 (2015).

[49] K. Bieniasz, M. Berciu, M. Daghofer, and A. M. Oleś, Phys. Rev. B 94, 085117 (2016).

[50] M. G. Yamada, M. Oshikawa, and G. Jackeli, Phys. Rev. Lett. 121, 097201 (2018).

[51] G. Khaliullin and S. Maekawa, Phys. Rev. Lett. 85, 3950 (2000).

[52] A. M. Oleś, Phys. Rev. B 28, 327 (1983).

[53] A. E. Antipov, I. S. Krivenko, V. I. Anisimov, A. I. Lichtenstein, and A. N. Rubtsov, Phys. Rev. B 86, 155107 (2012).

[54] For more details see in the Appendix-Supplemental Material.

[55] T. Mizokawa and A. Fujimori, Phys. Rev. B 51, 12880(R) (1995); 54, 5368 (1996); 56, R493 (1997).

[56] T. Mizokawa, D. I. Khomskii, and G. A. Sawatzky, Phys. Rev. B 60, 7309 (1999); 61, R3776 (2000); 61, 11263 (2000).
[57] T. Mizokawa, L. H. Tjeng, G. A. Sawatzky, G. Ghiringhelli, O. Tjernberg, N. B. Brookes, H. Fukazawa, S. Nakatsuji, and Y. Maeno, Phys. Rev. Latt. 97, 077202 (2001).

[58] H.-J. Noh, S.-J. Oh, B.-G. Park, J.-H. Park, J.-Y. Kim, H.D. Kim, T. Mizokawa, L. H. Tjeng, H.-J. Lin, C. T. Chen, S. Schuppler, S. Nakatsuji, H. Fukazawa, and Y. Maeno, Phys. Rev. B 72, 052411 (2005).

[59] See discussion of Fig. 20(d) in Ref. [43].

[60] A. M. Oleś, G. Khaliullin, P. Horsch, and L. F. Feiner, Phys. Rev. B 72, 214431 (2005).

[61] G. Khaliullin and P. Horsch, Phys. Rev. B 47, 463 (1993).

[62] R. Saha, F. Fauth, V. Caignaert, and A. Sundaresan, Phys. Rev. B 95, 184107 (2017).

[63] C. Domb and N. W. Dalton, Proc. Phys. Soc. 89, 859 (1966); Ł. Kurzawski and K. Malarz, Rep. Math. Phys. 70, 163 (2012). 